\title{
Paradigms in multiple sclerosis: time for a change, time for a unifying concept
}

\author{
Bernd Krone · John M. Grange
}

Received: 8 January 2011/Accepted: 9 April 2011/Published online: 6 May 2011

(c) The Author(s) 2011. This article is published with open access at Springerlink.com

\begin{abstract}
It has recently been suggested that, rather than being an autoimmune disease, multiple sclerosis (MS) is an example of a neurocristopathy, a pathological process resulting from a faulty development of the neural crest. Whilst several characteristics of the disease suggest a neurocristopathy, other aetiological factors require consideration, including hygiene-related factors that alter the immune responses to common pathogens resulting in an eclipse of immune reactivity that could protect against MS, the possible role of human endogenous retroviruses (HERVs) in pathogenesis and autoimmune phenomena, HLA polymorphism, vitamin D levels before and after birth and immune repair mechanisms. A postulated aetiological factor in MS, associated with altered vitamin D metabolism and abnormal HERV expression, is a longlasting disturbed redox regulation in the biosynthesis of a melanoma-like melanin pigment. Although intensive further studies on melanin pigments in nerve tissue in MS are required, the known properties of a pathological form of such pigments in melanoma could explain a number of observations in MS, including the impact of light, UV-light, and vitamin D, and could explain the clinical
\end{abstract}

\author{
B. Krone \\ Institute of Virology, Centre for Hygiene and Human \\ Genetics, University of Göttingen, Kreuzbergring 57, \\ 37075 Göttingen, Germany \\ Present Address: \\ B. Krone \\ Laboratory Medicine Institute Kassel Dr. Staber and Colleagues, \\ Druseltalstraße 61, 34131 Kassel-Wilhelmshöhe, Germany
}

J. M. Grange $(\square)$

Advanced Therapies Centre B2, London Clinic Cancer Centre,

22 Devonshire Place, London W1G 6JA, UK

e-mail: aya.sufya@btinternet.com manifestations of MS on the basis of an oscillating process of oxidative charge and discharge of the pigments and a threshold phenomenon with a change of the quasi-catalytic function of the pigment from destroying reactive oxygen radicals or species to transforming them to more harmful long-persisting highly reactive species. Taken together with the consequences of an adaptive process in partly demyelinated neurons, resulting in an increase in number of mitochondria, and the impact of stressful life events, these conditions are necessary and sufficient to explain the disease process of MS with its spatial (plaques) and temporal (attacks and remissions) characteristics. This suggested unifying concept of the pathogenesis of MS may open perspectives for prevention, diagnosis and therapy. In particular, prevention may be achieved by vaccinating against Epstein-Barr virus in early childhood.

Keywords Multiple sclerosis - Neurocristopathy ·

Endogenous retrovirus - Hygiene hypothesis .

Immune modulation $\cdot$ Autoimmunity $\cdot$ Redox regulation

\section{Introduction}

In a recent article published in this journal, Behan and Chaudhuri (2010) criticise the current paradigm that multiple sclerosis (MS) is primarily an autoimmune disorder and they cite data supporting a concept of this disease being a neurocristopathy; namely, a pathological process resulting from a faulty development of the neural crest. We fully agree that a reappraisal of the cause and nature of MS is overdue but there are no less than seven major aspects that are of critical relevance to such a reappraisal. These are exogenous infections, human endogenous retrovirus (HERV) activity, HLA polymorphism, immune repair, 
autoimmunity, vitamin D levels and cell damage by reactive oxygen species or radicals (ROS). As Behan and Chaudhuri rightly remark, the concept of a neurocristopathy has been more or less neglected but so has immune repair, whilst aspects such as HERVs have been considered largely in isolation from the other factors including the nature and timing of exogenous infections. The time has come to attempt to draw all the known aspects of this complex and puzzling disease together in the hope of paving the way to a unifying concept.

It is clear that the aetiology of MS is extremely complex as many co-factors may interact in different ways so that any single factor may appear to have a strong, weak or even antagonistic effect on the risk and course of the disease. In this context, features associated with MS may be necessary or merely enhancing or predisposing and, if necessary, may or may not be sufficient to explain the clinical manifestations of the disease. The challenge is to understand the roles of, and the interconnections between, these factors. Focusing on any single factor may yield conflicting data and, in general, the observed effects of single factors in conditions with complex aetiologies are much weaker than in those with a single cause. Large studies are therefore needed to reveal significant associations, and in smaller studies a non-significant association does not rule out a causative role.

A major challenge in MS is to understand the influences of exposure to visible and UV-light, of the significance of vitamin D levels, and of the supposed core-aspect of 'neurocristopathy', namely, melanin. Moreover, a unifying concept of MS must be able to give a simple explanation for the spatial distribution of the disease (in plaques) and the time course (attacks, remissions and demyelinating events).

\section{The hygiene hypothesis and Darwinian medicine}

A useful starting point in an endeavour to unravel the complexities of MS is the emerging discipline of 'Darwinian medicine' (Rook 2009; Krone and Grange 2010) with its central concept that hygiene-related factors in the industrialised nations increasingly isolate the human population from micro-organisms, both pathogens and, probably more importantly, commensals, that are crucial to the establishment of beneficial protective immunoregulatory networks. This concept, sometimes termed the hygiene hypothesis, has been advanced as an explanation for the considerable rise in the incidence of diseases associated with chronic inflammation mediated by immune dysregulation, notably allergies, asthma, vasculitis, autoimmune disorders, and some forms of cancer. Epidemiological studies clearly indicate that MS must be numbered amongst these environment-related conditions (Fleming and Fabry 2007), and would explain why MS occurs more often in the northern, industrialised, regions and why those migrating at an early age to a low-risk region take on the lower risk and those migrating at a later age to a high-risk region do not take on the higher risk.

\section{A possible role for the Epstein-Barr virus in the aetiology of MS}

A meta-analysis has established that more than $95 \%$ of all patients with MS have serological evidence of prior Epstein-Barr virus (EBV) infection, compared to $87 \%$ of control subjects (Santiago et al. 2010). Furthermore, there is a lag phase of several years or a decade or more between EBV infection and the clinical manifestation of MS (Haahr et al. 2004). During this rather long time period, EBVspecific antibody may well have dropped below detectable levels in some persons. Also, taking into account the distribution of specific antibody concentrations in the study groups, it is not only possible but also indeed likely that there are virtually no EBV-naïve persons in the patient group and, accordingly, past EBV infection has been postulated as a necessary condition for development of MS (Krone et al. 2008, 2009; Krone and Grange 2011; Pohl et al. 2006).

There are a number of hypothetical mechanisms by which EBV might induce MS (Ascherio and Bar-Or 2010; Korn 2008; Perron et al. 2009; Pohl 2009), though none of these convincingly explain the pathogenesis of the disease and it appears more likely that other infections synergize with EBV to produce a dysregulated immune response years or a decade before the clinical onset of MS.

One consequence of improved hygiene in the wealthier industrialised nations is that certain infections that were previously regularly encountered in infancy now occur at a much later time in life and after other infections may have altered the patterns of immune responses elicited by the former. Infection by EBV is a good example and it has been postulated that infections by various other pathogens bearing cross-reacting epitopes acquired before EBV may affect immunoregulatory networks, thereby leading to an attrition or eclipse of those regulatory T-cells (Tregs) that would otherwise protect against MS (Krone et al. 2009). Accordingly, a critical determinant of MS risk could be a compromised number or activity of protective Tregs (Levin et al. 2005; Brudek et al. 2009).

In this context, T-cells recognising the EBNA-1 protein of EBV play a central immune protective role in the control of EBV infection in healthy individuals although only a few of the many epitopes of EBNA-1 are recognised by $\mathrm{T}$-cells. By contrast, very many more are recognised in MS 
(Lünemann et al. 2006), and this so-called 'epitope spreading', probably induced by prior infections by pathogens bearing many cross-reactive epitopes, such as HHV-6 and Chlamydia pneumoniae, could divert immune responses away from protective ones.

\section{Endogenous retroviruses}

Human endogenous retroviruses have entered the human germ line successively over millions of years (Christensen et al. 2007; Perron and Lang 2010; Dolei and Perron 2009), and are present in the genome of all host cells. Most HERVs are defective, having been inactivated by negative selection and an accumulation of mutations (deletions, termination codons and frame shifts) and thus most of their genetic loci are inactive (Christensen 2005, 2010). Some reading frames have, however, remained open and code for complete proteins, such as the ERVWE1 locus on chromosome 7q21 which codes for syncytin-1, and one member of the HERV-W family, the MS-related virus (MSRV), can form complete virions under certain circumstances (Mameli et al. 2007). Moreover, it is well established that certain exogenous human retroviruses, HTLV-I and HIV, as well as Endogenous retroviruses in other species, such as rodents, ovines, and birds, can be cause of neurological and malignant diseases (Weiss 2006).

The expression of ERVWE1/MSRV in brain tissue from MS patients has been reported (Antony et al. 2007a, b; Sotgiu et al. 2002), and the number of such expressed sequences in the CSF has been shown to be higher in MS than in controls (Mameli et al. 2007; Sotgiu et al. 2006; Mameli et al. 2009). In addition, increased levels of antibody reactivity to specific retroviral HERV Gag and Env epitopes have been found in serum and CSF from MS patients (Christensen 2005), and these antibody levels are related with the activity of the disease (Christensen et al. 2007). The HERV-W encoded Env protein (ERVWE1, syncytin) is upregulated in glial cells in active MS lesions and, when expressed as a construct in astrocytes, has been shown to induce oligodendrocyte cell death via redoxreactants (Antony et al. 2004), as well as inducing an autoimmune cascade (Perron and Lang 2010).

Despite 20 years research, the role of HERVs as pathogens is still controversial but, nevertheless, they are the leading candidates for a link between genetic predisposition and environmental factors in causing a disease such as MS.

A potentially important link, one requiring experimental verification, between HERV-related phenomena and the pathogenesis of a neurocristopathy leading to MS involves the metabolism of melanin. This link is suggested by parallel observations in melanoma which have led to the concept that over-expression of certain HERV-encoded proteins results in metabolic changes that induce the formation of pathological forms of melanin (Krone and Grange 2010, 2011). Melanoma-melanin is very long lived and tends to become oxidatively charged and can therefore catalyse the formation of harmful reactive oxygen species over a long time span, in contrast to normal melanin which destroys such species (Tolleson 2005). Cells containing abnormal melanin are, accordingly, vulnerable to various forms of oxidative stress that, through damage to proteins, lipids and chromosomes, pave the way to cell degeneration or malignant transformation. By analogy, it is possible that expression of ERVWE1 proteins induce the biosynthesis of a melanoma-like melanin. In common with MS, the risk of developing melanoma is affected by a complex infectious background leading to the development of populations of T-cells that recognise a HERV-encoded envelope epitope, HERV-K-MEL (Krone and Grange 2010). In considering such parallels, it should be noted that melanomas develop from cells derived from the neural crest.

\section{Vitamin D}

Amongst the several environmental risk factors for MS (Ascherio and Munger 2007), a leading one is a low level of bioactive vitamin D3, but it is still controversial whether any and, if so, which of the many forms and actions of this vitamin is critical for MS (Szodoray et al. 2008; Smolders 2011). Thus, although vitamin D supplementation induced anti-inflammatory cytokine production with therapeutic benefit in MS patients (Smolders et al. 2010), immune modulating activity of this vitamin does not explain the risk-enhancing effect of low vitamin D3 levels which appear to commence during early in utero development as persons born in November/December have the lowest MS risk whilst those born in May/June have a highest risk. This difference is statistically significant (Willer et al. 2005). The effect was also seen in a small group of German patients with onset of MS in childhood (Fig. 1. Data supplied by courtesy of Hanefeld 2011).

It is suggested that low levels of this vitamin cause, via $\gamma$-glutamyl-transpeptidase, a reduction in the intra-cellular levels of glutathione, as has been demonstrated in rat astrocytes (Garcion et al. 1999), and a restricted capacity to reduce reactive oxygen species. The long-lasting impact of this effect may result from a favouring of the peroxide driven biosynthesis of melanoma-like melanin and by the long-living nature of the polymeric melanin redox pigments including pro-oxidative variants. As a consequence, more quinone/hydroquinone subunits of the pigment tend to be brought into the quinone form and, accordingly, the pigment takes on a pro-oxidative charge that changes the 


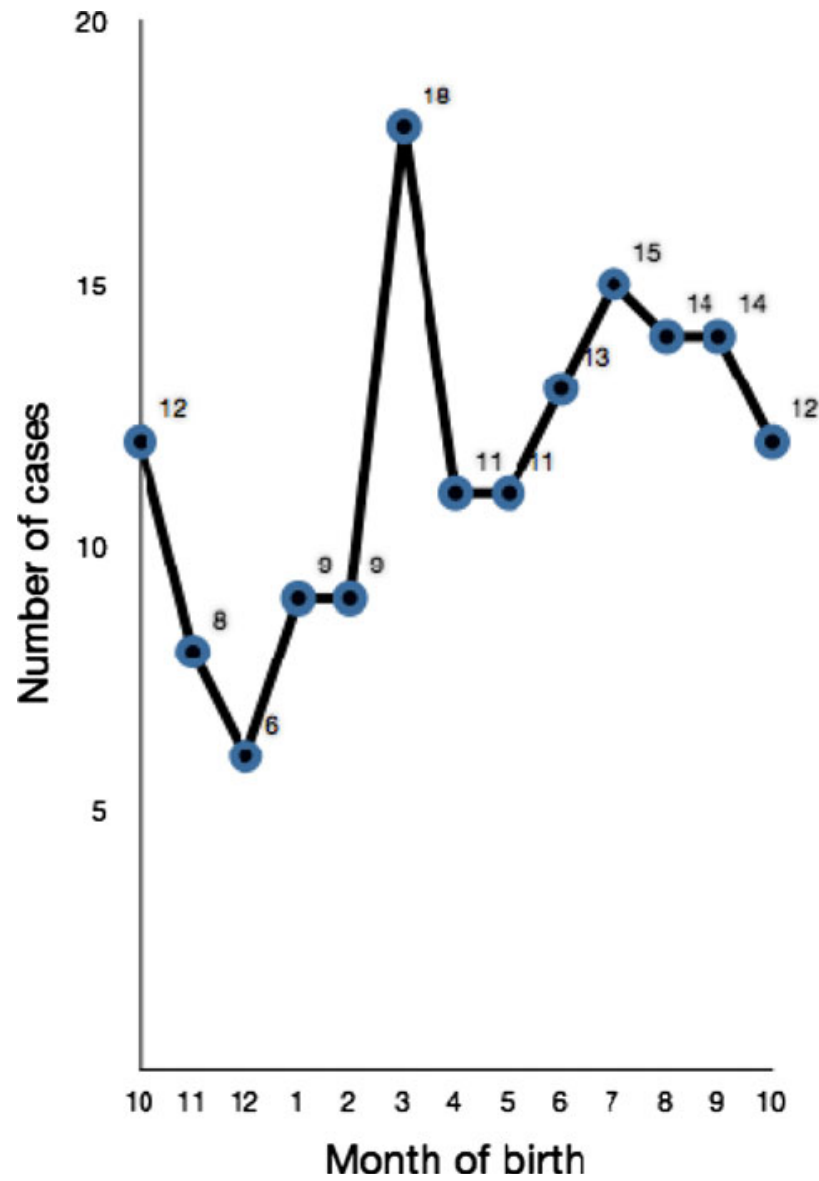

Fig. 1 Seasonal MS-risk

mode of its action from destroying short-living reactive oxygen species and radicals to transforming them into long living and more harmful ones. (Furthermore the pathological pigment can become reduced to a more or less normally functioning one, although it remains at risk of becoming pro-oxidatively charged again). It should be noted that the charging and discharging of the pigment is an oscillating process and that the change in the mode of action of the pigment in relation to pro-oxidative charge is a threshold phenomenon.

\section{HLA polymorphism}

Family studies indicate that genetic factors are involved in the risk of developing MS. Thus, for example, in northern countries with an overall lifetime MS risk of $0.1-0.2 \%$, the risk in identical twins and first, second and third degree relatives (with 100, 50, 25 and $12.5 \%$ genetic similarity, respectively) are $25,2-5,1-2$, and $<1 \%$, so that, at most, penetrance of the risk is only $25 \%$ (Goodin 2010). The strongest genetic risk factor is within the HLA-DRB1 locus and, more specifically, the HLA-DRB $1 * 1501$ allele although only a small minority of those with this allele develop MS, suggesting that many other genetic loci are involved in the determination of risk. Thus, the development of MS almost certainly depends on the combination of genetic susceptibility and environmental factors, notably EBV infection, vitamin D levels, and hygiene-related factors (Goodin 2009). In principle, HLA haplotypes and other genetic loci could affect risk by determining the repertoire of microbial epitopes presented to effector cells of the immune system and the levels and metabolism of vitamin D (Ramagopalan et al. 2009).

\section{Melanin, oxygen and mitochondrial damage}

As discussed above, two factors appear to be involved in the biosynthesis of a melanoma-like melanin, HERVrelated activity and low vitamin D levels. It seems that the two act synergistically and that they must even combine to produce the disease. Most of the vitamin D in humans is synthesised in the uncovered skin upon exposure to bright sunlight rich in UV-B. Reduced exposure to such light is related to geographical latitude and, when this is experienced during first trimester in pregnancy, it already initiates in utero a chain of events leading to the synthesis of a very long-living melanoma-like melanin. The hygienerelated factors leading to low intracellular glutathione peroxidase levels may well synergise with low vitamin D in inducing abnormal melanin synthesis and the subsequent regulation of its functional activity.

The biosynthesis of the melanoma-like pigment in conditions of low vitamin D levels would occur years or decades before the clinical onset of MS. Within the nerve cell, low glutathione peroxidase and low levels of its substrate glutathione result in diminished ability to reduce hydrogen peroxide and lipid peroxides. The peroxides react with the ortho-quinone structure of melanin subunits and its precursors and cleave the bond between the two carbonyl-groups of some of the ortho-quinone subunits thus generating a melanoma-like melanin (Ito and Wakamatsu 2008).

A switch to the pro-oxidative form of melanin is dependent on recent low vitamin D levels in combination with the generation of reactive oxygen species and radicals by stressful life events. In this context, it was shown in an Australian case-control study that past and current sun exposure and serum vitamin D levels are independently associated with a reduced risk of first demyelinating events in the central nervous system (Lucas et al. 2011), suggesting that vitamin $\mathrm{D}$ is involved in the biosynthesis of the pigment and on its switching to the pro-oxidative form.

Another factor affecting the biological activity of melanins is the hormone melatonin which causes aggregation 
of melanosomes within the cells during the night. This may explain why manifestation of MS is observed more frequently during the light months of the year with shorter nights (Meier et al. 2010).

The concepts reviewed above shed light on the observations that exacerbations of MS are dependent on oxidative/nitrosative stress and a diverse range of stressful life events (Mohr et al. 2004). Furthermore, the association between smoking and exacerbations of MS (Healy et al. 2009) could be related to the ability of nicotine to accumulate in melanin-containing cells and to affect melanin metabolism (Yerger and Malone 2006).

Molecular oxygen, which is highly reactive, is essential for all human cells, but in particular for nerve cells as these have no alternative ways of producing energy and, in the absence of oxygen, will die within a few minutes and are not replaced. Although nerve cells account for only about $2 \%$ of the body mass they consume (when body is at rest) about $20 \%$ of all available oxygen. Oxygen-based metabolic processes generate reactive oxygen species which are normally rapidly destroyed by enzymes such as catalases and glutathione peroxidises, processes requiring reduction substrates, in particular, glutathione. Another important protective factor is the melanin pigment, an important component of the cellular microenvironment that determines the half life of ROS and which is the last barrier against the harmful ROS when the first lines of protection, the endogenous antioxidant enzymes and free glutathione, are overrun. There is increasing evidence that ROS play a key role in the nerve damage in MS and other neurodegenerative and neuroinflammatory disorders, and the possible therapeutic use of anti-oxidant agents has been discussed (Gilgun-Sherki et al. 2004; van Horssen et al. 2011).

ROS are predominantly produced in the mitochondria as an unwanted side effect of endoxidation, oxidative phosphorylation and generation of ATP (Toyomizu et al. 2010). Accordingly any disturbance in mitochondrial function could have adverse, even fatal, consequences for irreplaceable nerve cells (Campbell and Mahad 2011). Increased numbers of mitochondria within nerve cells have been observed in active and inactive MS lesions and are presumed to be the result of adoptive, principally protective processes (Witte et al. 2009). Moreover, it is known that diseases of mitochondria are associated with degenerative neurological disease, particularly, those characterised by demyelination (Mahad et al. 2008). In this context, ROS causes damage to axons, resulting in demyelination (Witte et al. 2009; Mao and Reddy 2010), with a reduction in the ability of the axons to conduct electrical impulses. Accordingly, the affected cells need more energy and the number of mitochondria increases though, when even more stressed, they produce many more ROS. The link between stress and formation of ROS is thus provided by the action of the mitochondria. On excessive energy demand, ROS production by mitochondria increases and this, together with a depression of a protective mechanism, the energy consuming inducible proton leak, leads to a higher mitochondrial membrane potential with further ROS production (Toyomizu et al. 2010). Thus, although intracellular protective mechanisms exist, if these are over-run, a massive over-production of ROS occurs and may in part explain the focal demyelinating lesions characteristic of MS.

A change from normal to melanoma-like melanin may well tip the balance between protection and ROS-mediated damage. In this context, although MS has long been regarded as a disease initially affecting white matter of the brain, recent studies indicate that the disease process commences in the grey, melanin-pigmented, brain tissue (Dalton et al. 2004; Geurts and Barkhof 2008).

\section{Autoimmune reactions in MS—cause or consequence?}

Despite considerable debate in the literature, the question of whether MS is an autoimmune disease remains unresolved. It appears likely, however, that autoimmunity is a secondary phenomenon and at least one cause could be that some HERV-encoded proteins act as superantigens, i.e. antigens that non-specifically mediate a wide range of antibody responses, including autoimmune ones (Conrad et al. 1997). In addition, as HERV proviruses are an established part of the human genome, HERV-expressed proteins can be regarded as 'self' so that any immune responses to them could be regarded as being autoimmune ones.

A further indication that there is an autoimmune component to MS is the clinical response to $\beta$-interferon which has been shown to suppress the differentiation of Th17 cells, a class of T-cells involved in many autoimmune processes (Zhang and Markovic-Plese 2010). Also, the antibody natalizumab appears to confer benefit in MS by preventing the transmigration of proinflammatory T-cells from the peripheral blood to the central nervous system (Kivisäkk et al. 2009). As the underlying characteristic of the various elements of MS appears to result from chronic inflammation secondary to immune dysregulation, an improved therapeutic strategy may be an adjuvant-based immune modulating agent (Bottasso et al. 2009).

\section{Cell repair}

The ability of immune reactions to lead to the killing of abnormal cells by, for example, cytotoxic T-cells and NK cells is well known, but much less attention has been 
devoted to immune-mediated cell repair, which is of importance in situations, such as the CNS, when conservation of cells is essential. One repair mechanism involves the direct transfer of gangliosides from leucocytes to target cells and, by mediating DNA methylation, they suppress or silence the expression of HERV-encoded genes (Krone and Grange 2010; Stengel et al. 2010).

\section{An emerging paradigm?}

The aspects of the pathogenesis of MS briefly summarised above emphasise that the subject is very complex and that there are still numerous gaps in our understanding and many loose threads that have not yet been woven together. Nevertheless, a picture is emerging of the predisposing factors and the chain of events that result in the clinical expression of this disease.

These may be summarised as follows:

- The risk of MS is dependent on the 'biography' of the immune system rather than on acquisition of a specific and unusual infection. This biography is strongly influenced by hygiene-related factors.

- This biography mediates an eclipse of MS protective self-specific immune reactions. Candidate peptides as possible relevant targets have been proposed on the basis of epidemiological observations and genetic data bank entries.

- The risk of MS is related to HLA types, suggesting that the HLA molecules of the patients have, compared to unaffected individuals, a reduced or absent ability to present the relevant protective peptides to the corresponding regulatory and effector T-cells.

- A likely form of immune reactivity protecting against MS is one that is mediated by self-specific CD8+ $\mathrm{T}$-cells that perform immune repair and thereby suppress genetic expression of potentially harmful HERV genes.

- There is increasing evidence that MS is a HERVrelated disease. Expression of HERV proteins, in particular, the ERVWE1-encoded envelope glycoprotein, induces a disturbed redox regulation in affected cells.

- The putative link between HERV expression and longlasting disturbed redox regulation is the biosynthesis of a melanoma-like melanin pigment that tends to become oxidatively charged and to catalyse the formation of long-persisting highly reactive radicals and oxygen species. Vitamin D levels and HLA-related regulation of this vitamin, before and after birth, may affect melanin metabolism.

- As superantigens, HERV antigens can also induce autoimmune reactions as secondary phenomena in MS.
It should be noted that these key elements form a chain of events which, with the possible exception of the last one, autoimmunity, lead eventually to the manifestation of clinical disease. On the other hand, although these key factors are collectively, though not individually, necessary, they must be considered in the context of a putative key element, namely a 'neurocristopathy', resulting from abnormal melanin metabolism.

Applying Occam's razor, the clinical manifestation of MS as a focal demyelinating disorder can be considered in the 'front scenery' of the disease as the consequence of three conditions-based on the factors outlined abovethat combine with stressful events in life. First, an oscillating reaction in which normal melanin and, to a greater extent, abnormal melanin tends to become pro-oxidatively charged, with this charge being normalised by the glutathione-based reactions described above. Such oscillating reactions, illustrated by the Belousov-Zhabotinskii reaction, occur in various biological situations (Quo and Wang 2008). Second, a threshold is encountered at which, instead of destroying ROS, the abnormal pigment transforms them into longer living and more harmful ones. Finally, when the cell undergoes metabolic stress as a result of excessive energy demand, mitochondrial dysfunction ensues and, in cells with high numbers of mitochondria, massive amounts of ROS are produced. If transformed to long-living ones, there is ensuing cell damage, in particular, demyelination.

In addition to critically reviewing current concepts in MS, Behan and Chaudhuri (2010) review the evidence that MS is associated with a higher incidence of other neurological diseases such as malignant glioma, glioblastoma multiforme, neurofibromatosis Type I, and hypertrophic peripheral neuropathy, all of which develop in cells derived from the same progenitor glial cells. They also point to the high incidence of multicentric origin of these diseases in patients with MS. A possible cause, in addition to those suggested by Behan and Chaudhuri, and especially of glial malignancies, is a widespread disturbance in gene expression and metabolic activity in these cells secondary to overexpression of HERVs. There is a definite association between HERV expression and many cancers and the various mechanisms by which HERV expression can lead to genetic instability resulting in malignant transformation have been reviewed (Romanish et al. 2010).

The steps, albeit speculative, towards a unifying concept of the pathogenesis of MS suggested here may open the perspective for immune prevention of this disease by vaccinating against EBV in early childhood (Krone et al. 2009). Furthermore, they give a rationale for the established diagnosis of MS based on patterns and levels of specific antibodies against commonly encountered pathogenic viruses in the central nervous system (Reiber et al. 2009) and for the prognostic value of the amount of 
expression of certain HERV-W RNA sequences in the brain (Antony et al. 2006; Mameli et al. 2009). They also point to an increased importance in early MS, or before the clinical onset of MS, of retinal examination to detect pathological changes attributable to altered melanin (Graham et al. 1989).

Furthermore, they provide pointers to the development of preventive and therapeutic strategies for MS based on the concept of a neurocristopathy, in particular, involving the biosynthesis and bioactivity of melanoma-like melanin redox pigments (Krone and Grange 2011), with putative critical importance for exacerbations of MS that are observed to be dependent on oxidative/nitrosative stress and a diverse range of stress reactions (Mohr et al. 2004). In particular, vitamin D and other anti-oxidative vitamins may play a greater therapeutic role in the future. The concept also sheds rationality on therapeutic attempts to remove heavy metal ions, in particular, mercury and iron, which are incorporated into the melanoma-like melanins and contribute to their tendency to become pro-oxidatively charged. In this context, the concept of a neurocristopathy is supported by magnetic resonance studies that reveal early metabolic changes, the occurrence of slow axonal changes rather than acute ones, in MS, and a dilatation in shape of perivascular spaces (Groeschel et al. 2006).

\section{Conclusions}

Behan and Chaudhuri (2010) have provided a stimulus to further discussion on the concept of 'neurocristopathy' in the pathogenesis of MS. In response to this stimulus, we have taken tentative steps towards a testable unifying hypothesis based on described exogenous and endogenous factors predisposing to MS and centred on the biosynthesis of a melanoma-like melanin. This, however, requires experimental verification by further studies. Whilst MS still tightly holds some of its mysteries, light is penetrating the darkness and we are convinced that the time has come when an interdisciplinary programme of research could reduce the current complexity to a simple testable unifying hypothesis and to a rational approach to the development of preventive and therapeutic strategies for this disease.

Open Access This article is distributed under the terms of the Creative Commons Attribution Noncommercial License which permits any noncommercial use, distribution, and reproduction in any medium, provided the original author(s) and source are credited.

\section{References}

Antony JM, van Marle G, Opii W, Butterfield DA, Mallet F, Yong VW, Wallace JL, Deacon RM, Warren K, Power C (2004) Human endogenous retrovirus glycoprotein-mediated induction of redox reactants causes oligodendrocyte death and demyelination. Nat Neurosci 7:1088-1095

Antony JM, Izad M, Bar-Or A, Warren KG, Vodjgani M, Mallet F, Power C (2006) Quantitative analysis of human endogenous retrovirus-W env in neuroinflammatory diseases. AIDS Res Hum Retroviruses 22:1253-1259

Antony JM, Zhu Y, Izad M, Warren KG, Vodjgani M, Mallet F, Power C et al (2007a) Comparative expression of human endogenous retrovirus-W genes in multiple sclerosis. AIDS Res Hum Retroviruses 23:1251-1256

Antony JM, Ellestad KK, Hammond R, Imaizumi K, Mallet F, Warren KG, Power C (2007b) The human endogenous retrovirus envelope glycoprotein, syncytin-1, regulates neuroinflammation and its receptor expression in multiple sclerosis: a role for endoplasmic reticulum chaperones in astrocytes. J Immunol 179:1210-1224

Ascherio A, Bar-Or A (2010) EBV and brain matter(s)? Neurology 74:1092-1095

Ascherio A, Munger KL (2007) Environmental risk factors for multiple sclerosis. Part II: noninfectious factors. Ann Neurol 61:504-513

Behan PO, Chaudhuri A (2010) The sad plight of multiple sclerosis research (low on fact, high on fiction): critical data to support it being a neurocristopathy. Inflammopharmacology 18:265-290

Bottasso O, Docena G, Stanford JL, Grange JM (2009) Chronic inflammation as a manifestation of defects in immunoregulatory networks-implications for novel therapies based on microbial products. Inflammopharmacology 17:193-203

Brudek T, Christensen T, Aagard L, Petersen T, Hansen HJ, MøllerLarsen A (2009) B cells and monocytes from patients with active multiple sclerosis exhibit increased surface expression of both HERV-H Env and HERV-W Env, accompanied by increased seroreactivity. Retrovirology 6:104-116

Campbell GR, Mahad DJ (2011) Mitochondria as crucial players in demyelinated axons: lessons from neuropathology and experimental demyelination. Autoimmune Diseases 2011: Article ID 262847

Christensen T (2005) Association of human endogenous retroviruses with multiple sclerosis and possible interactions with herpes viruses. Rev Med Virol 15:179-211

Christensen T (2010) HERVs in neuropathogenesis. J Neuroimmune Pharmacol 5:326-335

Christensen T, Petersen T, Thiel S, Brudek T, Ellermann-Eriksen S, Møller-Larsen A (2007) Gene-environment interactions in multiple sclerosis: innate and adaptive immune responses to human endogenous retroviruses and herpes virus antigens and the lectin complement activation pathway. J Neuroimmunol 183:175-188

Conrad B, Weissmahr RN, Böni J, Arcari R, Schüpbach J, Mach B (1997) A human endogenous retroviral superantigen as candidate autoimmune gene in type I diabetes. Cell 90:303-313

Dalton CM, Chard DT, Davies GR, Miszkiel KA, Altmann DR, Fernando K, Plant GT, Thompson AJ, Miller DH (2004) Early development of multiple sclerosis is associated with progressive grey matter atrophy in patients presenting with clinically isolated syndromes. Brain 127:1101-1107

Dolei A, Perron H (2009) The multiple sclerosis-associated retrovirus and its HERV-W endogenous family: a biological interface between virology, genetics, and immunology in human physiology and disease. J Neurovirol 15:4-13

Fleming J, Fabry Z (2007) The hygiene hypothesis and multiple sclerosis. Ann Neurol 61:85-88

Garcion E, Sindji L, Leblondel G, Brachet P, Darcy F (1999) 1,25dihydroxyvitamin D3 regulates the synthesis of gamma-glutamyl transpeptidase and glutathione levels in rat primary astrocytes. J Neurochem 73:859-866 
Geurts JJ, Barkhof F (2008) Grey matter pathology in multiple sclerosis. Lancet Neurol 7:841-851

Gilgun-Sherki Y, Melamed E, Offen D (2004) The role of oxidative stress in the pathogenesis of multiple sclerosis: the need for effective antioxidant therapy. J Neurol 251:261-268

Groeschel S, Chong WK, Surtees R, Hanefeld F (2006) VirchowRobin spaces on magnetic resonance images: normative data, their dilatation, and a review of the literature. Neuroradiology 48:745-754

Goodin DS (2009) The causal cascade to multiple sclerosis: a model for MS pathogenesis. PLoS One 4:e4565

Goodin DS (2010) The genetic basis of multiple sclerosis: a model for MS susceptibility. BMC Neurol 10:101

Graham EM, Stanford MR, Sanders MD, Kasp E, Dumonde DC (1989) A point prevalence study of 150 patients with idiopathic retinal vasculitis: 1. Diagnostic value of ophthalmological features. Br J Ophthalmol 73:714-721

Haahr S, Plesner AM, Vestergaard BF, Höllsberg P (2004) A role of late Epstein-Barr virus infection in multiple sclerosis. Acta Neurol Scand 109:270-275

Hanefeld F (2011) Data from a German cohort with onset of MS in childhood, 1985 to 2002. Personal communication

Healy BC, Ali EN, Guttmann CR, Chitnis T, Glanz BI, Buckle G, Houtchens M, Stazzone L, Moodie J, Berger AM, Duan Y, Bakshi R, Khoury S, Weiner H, Ascherio A (2009) Smoking and disease progression in multiple sclerosis. Arch Neurol 66:858-864

Ito S, Wakamatsu K (2008) Chemistry of mixed melanogenesispivotal roles of dopaquinone. Photochem Photobiol 84:582-592

Kivisäkk P, Healy BC, Viglietta V, Quintana FJ, Hootstein MA, Weiner HL, Khoury SJ (2009) Natalizumab treatment is associated with peripheral sequestration of proinflammatory $\mathrm{T}$ cells. Neurology 72:1922-1930

Korn T (2008) Pathophysiology of multiple sclerosis. J Neurol 255(Suppl 6):2-6

Krone B, Grange JM (2010) Melanoma, Darwinian medicine and the Inner world. J Cancer Res Clin Oncol 136:1787-1794

Krone B, Grange JM (2011) Multiple sclerosis-are protective immune mechanisms compromised by a complex infectious background? Autoimmune Dis 2011: Article ID 708750

Krone B, Pohl D, Rostasy K, Kahler E, Brunner E, Oeffner F, Grange JM, Gärtner J, Hanefeld F (2008) Common infectious agents in multiple sclerosis: a case-control study in children. Mult Scler $14: 136-139$

Krone B, Oeffner F, Grange JM (2009) Is the risk of multiple sclerosis related to the 'biography' of the immune system? J Neurol 256:1052-1060

Levin LI, Munger KL, Rubertone MV, Peck CA, Lennette ET, Spiegelman D, Ascherio A (2005) Temporal relationship between elevation of Epstein-Barr virus antibody titers and initial onset of neurological symptoms in multiple sclerosis. JAMA 293:2496-2500

Lucas RM, Ponsonby AL, Dear K, Valery PC, Pender MP, Taylor BV, Kilpatrick TJ, Dwyer T, Coulthard A, Chapman C, van der Mei I, Williams D, McMichael AJ (2011) Sun exposure and vitamin D are independent risk factors for CNS demyelination. Neurology 76:540-548

Lünemann JD, Edwards N, Muraro PA, Hayashi S, Cohen JI, Münz C, Martin R (2006) Increased frequency and broadened specificity of latent EBV nuclear antigen-1-specific T cells in multiple sclerosis. Brain 129:1493-1506

Mahad D, Lassmann H, Turnbull D (2008) Review: mitochondria and disease progression in multiple sclerosis. Neuropathol Appl Neurobiol 34:577-589

Mameli G, Astone V, Arru G, Marconi S, Lovato L, Serra C, Sotgiu S, Bonetti B, Dolei A (2007) Brains and peripheral blood mononuclear cells of multiple sclerosis (MS) patients hyperexpress MS-associated retrovirus/HERV-W endogenous retrovirus, but not human herpesvirus 6. J Gen Virol 88:264-274

Mameli G, Poddighe L, Astone V, Delogu G, Arru G, Sotgiu S, Serra C, Dolei A (2009) Novel reliable real-time PCR for differential detection of MSRVenv and syncytin-1 in RNA and DNA from patients with multiple sclerosis. J Virol Methods 161:98-106

Mao P, Reddy PH (2010) Is multiple sclerosis a mitochondrial disease? Biochim Biophys Acta 1802:66-79

Meier DS, Balashov KE, Healy B, Weiner HL, Guttmann CR (2010) Seasonal prevalence of MS disease activity. Neurology 75:799-806

Mohr DC, Hart SL, Julian L, Cox D, Pelletier D (2004) Association between stressful life events and exacerbation in multiple sclerosis: a meta-analysis. Br Med J 146:144-152

Perron H, Lang A (2010) The human endogenous retrovirus link between genes and environment in multiple sclerosis and in multifactorial diseases associating neuroinflammation. Clin Rev Allergy Immunol 39:51-61

Perron H, Bernard C, Bertrand JB, Lang AB, Popa I, Sanhadji K, Portoukalian J (2009) Endogenous retroviral genes, herpesviruses and gender in multiple sclerosis. J Neurol Sci 286:65-72

Pohl D (2009) Epstein-Barr virus and multiple sclerosis. J Neurol Sci 286:62-64

Pohl D, Krone B, Rostasy K, Kahler E, Brunner E, Lehnert M, Wagner HJ, Gärtner J, Hanefeld F (2006) High seroprevalence of Epstein-Barr virus in children with multiple sclerosis. Neurology 67:2063-2065

Quo CF, Wang MD (2008) Quantitative analysis of numerical solvers for oscillatory biomolecular system models. BMC Bioinformatics 9(Suppl 6):S17

Ramagopalan SV, Maugeri NJ, Handunnetthi L, Lincoln MR, Orton SM, Dyment DA, Deluca GC, Herrera BM, Chao MJ, Sadovnick AD, Ebers GC, Knight JC (2009) Expression of the multiple sclerosis-associated MHC class II Allele HLA-DRB1*1501 is regulated by vitamin D. PLoS Genet 5:e1000369

Reiber H, Teut M, Pohl D, Rostasy KM, Hanefeld F (2009) Paediatric and adult multiple sclerosis: age-related differences and time course of the neuroimmunological response in cerebrospinal fluid. Mult Scler 15:1466-1480

Romanish MT, Cohen CJ, Mager DL (2010) Potential mechanisms of endogenous retroviral-mediated genomic instability in human cancer. Semin Cancer Biol 20:246-253

Rook GA (ed) (2009) The hygiene hypothesis and Darwinian medicine. Birkhäuser, Basel

Santiago O, Gutierrez J, Sorlozano A, de Dios Luna J, Villegas E, Fernandez O (2010) Relation between Epstein-Barr virus and multiple sclerosis: analytic study of scientific production. Eur J Clin Microbiol Infect Dis 29:857-866

Smolders J (2011) Vitamin D and multiple sclerosis: correlation, causality, and controversy. Autoimmune Dis 2011:629538

Smolders J, Peelen E, Thewissen M, Cohen Tervaert JW, Menheere P, Hupperts R, Damoiseaux J (2010) Safety and T cell modulating effects of high dose vitamin D3 supplementation in multiple sclerosis. PLoS One 5:e15235

Sotgiu S, Serra C, Mameli G, Pugliatti M, Rosati G, Arru G, Dolei A (2002) Multiple sclerosis-associated retrovirus and MS prognosis: an observational study. Neurology 59:1071-1073

Sotgiu S, Arru G, Mameli G, Serra C, Pugliatti M, Rosati G, Dolei A (2006) Multiple sclerosis-associated retrovirus in early multiple sclerosis: a six-year follow-up of a Sardinian cohort. Mult Scler 12:698-703

Stengel S, Fiebig U, Kurth R, Denner J (2010) Regulation of human endogenous retrovirus- $\mathrm{K}$ expression in melanomas by $\mathrm{CpG}$ methylation. Genes Chromosomes Cancer 49:401-411 
Szodoray P, Nakken B, Gaal J, Jonsson R, Szegedi A, Zold E, Szegedi G, Gesztelyi R, Brun JG, Zeher M, Bodolay E (2008) The complex role of vitamin D in autoimmune diseases. Scand $\mathbf{J}$ Immunol 68:261-269

Tolleson WH (2005) Human melanocyte biology, toxicology, and pathology. J Environ Sci Health C Environ Carcinog Ecotoxicol Rev 23:105-161

Toyomizu M, Kikusato M, Amo T, Azad MAK (2010) Mitochondrial energetics and ROS production in chicken skeletal muscle: application of modular kinetic analysis. In: Vrovetto GM (ed) Energy and protein metabolism and nutrition. EAAP publication No. 127. Wageningen Academic Publishers, The Netherlands, pp 39-46

van Horssen J, Witte ME, Schreibelt G, de Vries HE (2011) Radical changes in multiple sclerosis pathogenesis. Biochim Biophys Acta 1812:141-150
Weiss RA (2006) The discovery of endogenous retroviruses. Retrovirology 3:67

Willer CJ, Dyment DA, Sadovnick AD, Rothwell PM, Murray TJ, Ebers GC, Canadian Collaborative Study Group et al (2005) Timing of birth and risk of multiple sclerosis: population based study. Br Med J 330:120-123

Witte ME, B $\varnothing$ L, Rodenburg RJ, Belien JA, Musters R, Hazes T, Wintjes LT, Smeitink JA, Geurts JJ, De Vries HE, van der Valk P, van Horssen J (2009) Enhanced number and activity of mitochondria in multiple sclerosis lesions. J Pathol 219:193-204

Yerger VB, Malone RE (2006) Melanin and nicotine: a review of the literature. Nicotine Tob Res 8:487-498

Zhang X, Markovic-Plese S (2010) Interferon beta inhibits the Th17 cell-mediated autoimmune response in patients with relapsingremitting multiple sclerosis. Clin Neurol Neurosurg 112:641-645 